\section{$\underset{\substack{\text { hommes } \\ \text { \& migrations }}}{ }$}

\section{Hommes \& migrations}

Revue française de référence sur les dynamiques

migratoires

$1309 \mid 2015$

Le $3 e$ âge des migrants

\title{
La transportabilité des prestations de sécurité sociale
}

Retraite, soins et minima sociaux

Jacques Wels, Nawal Bensaid et Florence Legros

\section{(2) OpenEdition \\ Journals}

Édition électronique

URL : http://journals.openedition.org/hommesmigrations/3087

DOI : 10.4000/hommesmigrations.3087

ISSN : 2262-3353

Éditeur

Musée national de l'histoire de l'immigration

\section{Édition imprimée}

Date de publication : 1 janvier 2015

Pagination : 107-115

ISBN : 978-2-919040-30-8

ISSN : 1142-852X

Référence électronique

Jacques Wels, Nawal Bensaid et Florence Legros, "La transportabilité des prestations de sécurité sociale », Hommes \& migrations [En ligne], 1309 | 2015, mis en ligne le 01 janvier 2017, consulté le 21 avril 2019. URL : http://journals.openedition.org/hommesmigrations/3087 ; DOI : 10.4000/ hommesmigrations.3087 


\title{
LA TRANSPORTABILITÉ DES PRESTATIONS DE SÉCURITÉ SOCIALE RETRAITE, SOINS ET MINIMA SOCIAUX
}

par JACQUES WELS, doctorant FNRS-FRFC à l'université libre de Bruxelles (Centre Metices), NAWAL BENSAID, doctorante FNRS à l'université libre de Bruxelles (Germe), et FLORENCE LEGROS, professeur des universités, université Paris-Dauphine.

\author{
Le vieillissement des populations migrantes pose la question \\ de la transportabilité de leurs retraites et de leurs soins \\ de santé entre le ou les pays dans lesquels ils ont acquis leurs \\ droits et leur pays d'origine. Des accords bilatéraux de Sécurité \\ sociale permettent de normaliser la gestion de cette épineuse \\ question du transfert des prestations sociales versées d'un pays \\ à l'autre aux migrants âgés. Une approche comparative entre \\ le Maroc, la France et la Belgique révèle un effort \\ de modernisation ces dix dernières années.
}

Les flux migratoires entre le Maroc et la Belgique, et entre le Maroc et la France, dépendent largement de processus ancrés dans des réalités historiques particulières à chacun des corridors migratoires. Ces particularités historiques ont fortement impacté les mouvements migratoires entre les pays et les cadres législatifs qui leur sont associés. En ce sens, les conventions bilatérales en matière de Sécurité sociale varient selon les corridors. Pour l'heure, le Maroc a signé 18 conventions de ce type (dont 14 sont actuellement appliquées), mais, si ces dernières ont en commun d'assurer la "portabilité du droit”, autrement dit la possibilité de jouir à l'étranger de la protection sociale d'un pays tiers, les droits qu'elles recouvrent ainsi que les modalités qui leur sont associées varient.

Notons par ailleurs que, pour l'heure, peu de travaux ont été produits sur la transportabilité des droits sociaux ${ }^{1}$ et particulièrement sur les corridors franco-marocain ${ }^{2}$ et belgo-marocain, ce dernier

1. Johanna Avato, "Portability of social security and health care benefits in Italy", in World Bank Background Paper, $n^{\circ}$ 6, 2008 ; Johanna Avato, "Definitions, good practices, and global estimates on the status of social protection for international migrants", in SP Discussion Paper, $n^{\circ}$ 51, 2009 ; Robert Holzmann, Johannes Koettl, Taras Chernetsky, "Portability regimes of pension and health care benefits for international migrants: an analysis of issues and good practices", Washington, D.C., World Bank, 2005 ; Robert Holzmann, Edward Palmer, "Pension Reform. Issues and Prospects for Non-Financial Defined Contribution (NDC) Schemes", Washington, D.C., World Bank, 2006, p. 671. 2. Ilham Dkhissi, Jean-Marc Dupuis Claire El Moudden, "Incidences de la convention franco-marocaine de Sécurité sociale sur les retraités CNAV nés et résidant au Maroc", in colloque "Vieillissement de la population dans les oays du Sud", Meknès, 17-19 mars 2011. 
n'ayant pour l'heure fait l'objet d'aucune étude approfondie. Par ailleurs, ces travaux ont été menés soit sous un angle théorique mettant en avant la nécessité de respecter le caractère assurantiel des régimes de protection sociale ${ }^{3}$, soit sous un angle statistique en observant les flux des pensions versées aux migrants retraités de retour dans leur pays d'origine $e^{4}$. Il est question ici d'examiner les conséquences de ces signatures de convention sur les flux migratoires et sur les prestations reçues ${ }^{5}$.

\section{France-Maroc, Belgique-Maroc : deux processus migratoires distincts}

La présence des Marocains en France et en Belgique est généralement considérée comme résultant des conventions bilatérales de main-d'œuvre signées respectivement en 1963 et 1964 . Cependant, des flux de migrants marocains en Belgique et en France avaient déjà été enregistrés, notamment durant les deux conflits mondiaux. La participation à l'effort de guerre constitue en cela un premier chemin migratoire. Dans les années 1950, l'arrivée massive de travailleurs marocains dans les deux pays vient changer le visage des migrations. De sporadique, le flux devient continu et sera progressivement encadré par des accords de main-d'œuvre signés entre les pays.

C'est principalement après la déclaration d'indépendance du Maroc en 1956 que l'immigration de masse débute, encadrée par les conventions bilatérales évoquées. 40000 Marocains migrent vers la
Belgique entre 1964 et 1974. En 1974, l'Europe ferme ses frontières à la main-d'œuvre étrangère et seule la migration pour regroupement familial reste possible ${ }^{6}$.

Cette décision va modifier radicalement le profil des migrants dans les deux pays : leur population, exclusivement masculine, va évoluer vers une population plus variée incluant les femmes et les enfants des travailleurs?

En raison des dispositifs en matière de regroupement familial, le flux ne s'est guère interrompu malgré les restrictions en matière de politique migratoire. En France en 2010, le nombre de Marocains s'élevait à environ 435000 personnes, soit le troisième groupe d'étrangers en France $(11,4 \%)$, après les Portugais (13,0 \%) et les Algériens (12,2 \%). Selon l'Institut national de la statistique et des études économiques (Insee), le nombre de migrants d'origine marocaine (migrants de seconde et troisième génération) s'élève en France à 1314000 personnes. Le flux de Marocains vers la France sélève lui à 24000 personnes en 2010, ce qui représente le flux d'étrangers le plus important après les Algériens.

Pour autant, et même si les effectifs de migrants marocains restent élevés, ils ont globalement chuté dans les deux pays à partir des années 1990. Cela étant, cette évolution n'est pas la conséquence d'un ralentissement des migrations, mais résulte de changements législatifs visant à faciliter les procédures de naturalisation des étrangers résidant légalement sur le territoire ${ }^{8}$. Si en Belgique, en 1999, 9133 acquisitions de la nationalité étaient enregistrées, elles étaient au nombre de 21917 en 2000 et de 24018 en 2001. En France, Denis Fougère et Mirna Safi ${ }^{9}$ ont montré que 23,7 \% des Marocains présents en France entre 1968 et 1999 ont été naturalisés. 
/// Tableau 1: Répartition des citoyens marocains de plus de 60 ans par sexe et par pays d'installation (2010)

\begin{tabular}{|c|c|c|c|c|c|}
\hline & \multicolumn{2}{|c|}{60 ANS ET PLUS } & \multirow{2}{*}{$\begin{array}{c}\% \text { DE LA } \\
\text { POPULATION } \\
\text { MAROCAINE } \\
\text { TOTALE }\end{array}$} & \multicolumn{2}{|c|}{$\begin{array}{c}\text { \% DE LA POPULATION MAROCAINE } \\
\text { TOTALE, PAR SEXE }\end{array}$} \\
\hline & HOMMES & FEMMES & & HOMMES & FEMMES \\
\hline FRANCE & 51,395 & 25,884 & $18 \%$ & $23 \%$ & $12 \%$ \\
\hline BELGIQUE & 4,385 & 6,455 & $13 \%$ & $10,75 \%$ & $15,70 \%$ \\
\hline
\end{tabular}

Source : STATBEL et Insee.

\section{Structure de la population marocaine et transfert des droits sociaux}

En s'intéressant plus spécifiquement aux groupes d'âge de nationalité marocaine concernés par la pension et par les droits sociaux qui leur sont associés (60 ans et plus), on recense en Belgique 6455 femmes et 4385 hommes de nationalité marocaine en 2010. Ces chiffres représentent $13 \%$ du total des citoyens marocains en Belgique. Il couvre $15,7 \%$ du total des femmes marocaines en Belgique et 10,75\% de celui des hommes. En France, pour la même période, ces chiffres sélèvent à 25884 femmes et à 51395 hommes de nationalité marocaine. Ils représentent $18 \%$ du total des citoyens marocains en France selon l'Insee en 2010, dont $12 \%$ pour les femmes et $23 \%$ pour les hommes (tableau 1 ).

Le nombre de bénéficiaires de pensions belges au Maroc semble assez faible (5 497), comparé au nombre de bénéficiaires dans les pays de l'Union européenne (147 794), dans le reste de l’Europe
(70 848) ou en Amérique du Nord et centrale (9947). En comparaison avec la Turquie (7 651), la France (40 507), l'Italie (37 716) et l'Espagne (20 689), le nombre de pensions versées au Maroc depuis la Belgique est également extrêmement faible selon l'ONP en 2013. Néanmoins, les dépenses mensuelles totales à destination du Maroc sont plus élevées qu'attendu : 4,25\% des dépenses totales sont consenties par la Belgique au payement des pensions.

Concernant les pensions transférées par la Caisse nationale d'assurance vieillesse (Cnav) française, le Maroc est le pays le plus concerné après l'Algérie, avec un total de 53139 individus en 2012. Le montant total s'élève à 318 millions d'euros en 2012, selon le Centre des liaisons européennes et internationales de sécurité sociale (CLEISS). Le Maroc est également le deuxième pays à percevoir les montants les plus élevés de pensions issues des caisses de retraite complémentaire (Association pour le régime de retraite complémentaire des salariés, Arrco, et Association générale des institutions de retraite des cadres, Agirc) avec un total de 33956 individus concernés pour 102 millions d'euros en 2012. Concernant, enfin, les pensions 
d'invalidité, le montant s'élève à 1,7 million d'euros en 2012 (deuxième pays de perception également). Ces transferts sont en outre corrélés à un marché du travail où la population marocaine est largement disqualifiée. En Belgique, le pourcentage d'inactifs dans la population marocaine en 2007 s'élève à 75,2 \% du total des femmes marocaines en Belgique et à 39,1\% du total des hommes. De la même façon, en France, le total de la population active marocaine en France sélève à 195775 personnes, parmi lesquelles on recense 57593 personnes sans emploi à la recherche d'un emploi, ce qui représente $29 \%$ de la population active de ce groupe.

De telles données invitent à penser que le phénomène de migration de retour n'a pas l'ampleur à laquelle on pouvait s'attendre. Bien que les chiffres présentés soient difficiles à traiter avec finesse, un rapide calcul permet

Le principe de réciprocité

s'applique à la pension

de retraite. Dans la mesure où les trois pays ont un système

de pension efficient, ces dernières sont transférables d'un pays à l'autre selon des modalités fixées par l'accord. d'éclairer la tendance : avec 435000 ressortissants marocains en France en 2010, le nombre de pensions de vieillesse françaises versées au Maroc n'était que de 53139 à la même date, soit un ratio de 8,2 individus sur 100. En Belgique, le nombre de ressortissants marocains en 2013 était de 86233 pour un nombre total de pensions de retraite payées au Maroc de 5497, soit un ratio de 15,7 individus sur 100. Ces chiffres sont, par ailleurs, à relativiser à la lumière du nombre d'acquisitions de nationalité dans les deux pays (près de $25 \%$ ), qui ont largement fait décroître la population de nationalité marocaine, mais également au regard du contenu des accords qui permettent aux individus qui ne sont ni de nationalité, ni d'origine marocaines de prendre leur retraite au Maroc. On assiste en cela à un réel "mythe du retour 10", dans la mesure où, si, qualitativement, la figure du migrant âgé retournant vivre au pays est relativement tenace, on n'observe pas un retour quantitativement aussi important que le mouvement d'arrivée.

\section{Les conventions bilatérales de sécurité sociale}

La première convention bilatérale de Sécurité sociale entre la Belgique et le Maroc a été signée en 1968, quatre ans après la ratification de la convention de main-d'œuvre. Cette convention a régi, hormis un certain nombre de modifications introduites ultérieurement par arrangements administratifs, la transférabilité des prestations sociales entre les deux pays pendant plus de quarante ans. Un nouveau projet de convention est cependant en voie de finalisation. L'accord bilatéral franco-marocain a, quant à lui, été signé le 9 juillet 1965, deux ans après la convention de main-d'œuvre. Celuici a, cependant, été remplacé par le nouvel accord de Sécurité sociale d'octobre 2011, déjà entré en vigueur. Pour l'heure, la transférabilité des prestations est régie par un accord modernisé dans le cas de la France et par l'accord initial, légèrement amélioré par arrangements administratifs, dans le cas de la Belgique. Les deux conventions reposent sur deux principes qui régissent, de manière plus générale, l'ensemble des conventions bilatérales signées entre les pays : la réciprocité du droit et la transférabilité des prestations contributives.

La réciprocité du droit fait référence à l'idée que toute prestation sociale transférable dans un sens l'est également dans l'autre sens. La portabilité d'une prestation est donc déterminée par l'existence réciproque du droit dans les deux pays concernés par l'accord. Dans la mesure où l'accord bilatéral couvre les flux migratoires dans les deux sens, ce dernier nécessite - sauf exception prévue un cadre commun de Sécurité sociale.

Le principe de réciprocité s'applique à la pension de retraite. Dans la mesure où les trois pays ont un système de pension efficient, ces dernières sont transférables d'un pays à l'autre selon des modalités fixées par l'accord. Dans le cas de l'accord belgo-marocain, la pension de retraite d'une 
personne ayant travaillé en Belgique, qui introduit une demande de retraite à la Belgique et part s'installer au Maroc, sera payée directement par l'Office national des pensions (ONP) belge, sans transiter par l'institution marocaine. Dans le cas où cette personne aurait une double carrière, au Maroc et en Belgique, l'institution auprès de laquelle la demande de retraite est introduite se charge de totaliser les périodes de travail sur son territoire et en dehors. Inversement, si la personne introduit sa demande au Maroc, c'est la Caisse nationale de sécurité sociale (CNSS) qui sera en charge de payer la pension de retraite, que la carrière ait été marocaine uniquement, double ou réalisée sur le sol belge ou français. Le cas français suit la même logique, en prévoyant le cas échéant la totalisation des périodes de travail prestées au Maroc. Cependant, contrairement à l'accord belgo-marocain, le versement de la pension par les institutions françaises ne se fait pas directement entre l'institution et l'individu, cette dernière transite en effet par la CNSS et sera versée directement par la CNSS.

\section{Les limites de la récriprocité du droit}

En matière de retraite, le cas des "pensions de survie" demeure problématique. La pension de survie permet au conjoint d'un individu décédé de bénéficier d'une pension relative au passé professionnel de l'individu et aux conditions de vie du conjoint. Néanmoins, si le dispositif existe au Maroc, c'est la définition juridique de la cessation du contrat de mariage qui pose problème. Au Maroc, deux systèmes de rupture du contrat de mariage coexistent: le divorce et la répudiation ${ }^{11}$. Dans la mesure où la répudiation s'avère être une procédure moins coûteuse que le divorce et ne nécessite pas la présence du conjoint, cette solution a largement été privilégiée. Or la Belgique et la France ne reconnaissent pas la répudiation comme une procédure légitime de rupture du contrat de mariage. Autrement dit, le remariage après répudiation peut être considéré en Belgique et en France comme un cas de polygamie, puisque la rupture n'est pas avérée dans le droit civil belge et français.

Ces cas - qui ne sont pas rares pour la population âgée - donnent lieu à une répartition de la pension de survie entre les différents conjoints. Cette répartition peut cependant être considérée comme illégitime par le ou les conjoints bénéficiaires de la pension de survie. Il n'est donc pas rare que des Bien que la réciprocité du droit soit une constante dans les accords bilatéraux, il existe, en fonction des accords, un certain nombre d'exceptions. cas de répartition de la pension de veuve après répudiation soient portés devant les tribunaux. En outre, d'un point de vue juridique, la non-reconnaissance de la répudiation en droit belge et français introduit en quelque sorte, par le biais des accords bilatéraux, la polygamie dans le droit civil belge et français. Cela donne lieu à une jurisprudence relativement diversifiée sur la question ${ }^{12}$. En 2003, cependant, le Code civil marocain (Mudawana) a été largement modifié. Si la répudiation n'est pas abolie par la réforme, certaines limites ont été fixées qui, à terme, produiront une baisse potentielle du nombre de cas.

Bien que la réciprocité du droit soit une constante dans les accords bilatéraux, il existe, en fonction des accords, un certain nombre d'exceptions. La convention conclue entre la France et le Maroc a été en ce sens initialement plus large que celle conclue avec la Belgique, en introduisant la portabilité du droit pour des prestations françaises ne trouvant pas leur équivalent au Maroc. Ce fut le cas, par exemple, pour les indépendants qui, non couverts au Maroc, ont pu bénéficier d'une prestation dans le cadre de la convention, ce qui n'est pas le cas pour l'accord belgo-marocain. De plus, la réciprocité du droit peut être limitée par des conditions posées par les États eux-mêmes, quand bien 
même la réciprocité serait assurée. C'est le cas, par exemple, pour les allocations familiales qui ont été limitées à un nombre d'enfants, en Belgique et en France.

De même, la question des soins de santé resté éminemment problématique. La convention bilatérale originale entre la Belgique et le Maroc n'incluait pas la couverture de soins de santé, mais certaines situations spécifiques avaient été néanmoins prévues indirectement par la convention et ont été implémentées par la suite, via des "arrangements administratifs". Ce fut le cas pour les soins de santé couvrant les séjours temporaires au Maroc et, plus récemment, pour les membres de la famille de personnes résidant au Maroc. Le $1^{\mathrm{er}}$ mars 2006, une assurance maladie obligatoire (AMO) entre en vigueur au Maroc. Cette dernière permet d'offrir la réciprocité du droit en matière de soins de santé. Mais l'accord belgomarocain initial ne prévoit nullement la couverture de santé des retraités. Elle n'a donc pu être implémentée par le seul moyen d'un arrangement administratif. Ce fut l'occasion, pour les autorités belges et marocaines, de négocier un nouvel accord introduisant la portabilité des soins de santé entre les deux pays. Un projet d'accord a été exposé à la presse en février 2014. L'accord franco-marocain a connu une évolution différente. La convention initiale prévoyait en effet la portabilité des soins de santé pour l'ensemble des catégories de populations à l'exception des retraités.

\section{Les prestations contributives}

Dans nos systèmes bismarckiens, les prestations contributives doivent être différenciées des prestations non contributives. Les premières sont accordées sur la base des cotisations sociales du travail, et la valeur du montant de la prestation est calculée
- dans des limites définies par le droit - au prorata des contributions perçues. Inversement, les prestations non contributives offrent un revenu universel qui ne varie pas et n'est pas accordé en fonction des cotisations prélevées sur le travail. Seules les prestations contributives, délivrées sur une base individuelle, sont couvertes par les accords bilatéraux. Les prestations non contributives - délivrées sur une base universelle et limitée au territoire national - ne sont quant à elles pas transportables.

Dans le cadre des deux accords bilatéraux, les prestations de type "minima sociaux" ne sont, compte tenu de leur inexistence, pas transportables. Les bénéficiaires d'un minimum vieillesse en France - rebaptisé "allocation de solidarité pour les personnes âgées" (Aspa) -, ainsi que les bénéficiaires d'une garantie de revenu aux personnes âgées (Grapa) en Belgique, ne peuvent pas bénéficier de telles prestations dans le cadre de l'accord bilatéral. La transportabilité du droit, pour ces deux types de prestations non contributives, est impossible. Cette situation génère trois cas de figure distincts. Dans un premier cas, l'individu bénéficiaire d'une prestation non contributive ne migre simplement pas vers le Maroc afin de continuer de percevoir le bénéfice de son allocation. Dans un second cas, la personne migre au Maroc et n'est plus en mesure de percevoir ce type de prestation. Enfin, un troisième cas, intermédiaire, permet la mobilité de la prestation sous réserve d'une résidence et d'une durée minimale de séjour dans le pays prestataire. Pour la Belgique comme pour la France, toute personne résidant plus de six mois par an en dehors du territoire national perd ses droits à la prestation. Il faut donc, pour conserver le droit à la prestation, effectuer un séjour de plus de six mois dans le pays qui délivre la prestation. Néanmoins, il est du seul ressort de l'organisme prestataire de contrôler l'effectivité de la résidence au pays. Cela laisse entrevoir une portabilité de fait des minima sociaux qui est largement fonction des contrôles effectués. En l'état, il est impossible de chiffrer le nombre de cas. 
Un résident chibani du foyer Adoma "Les Grésillons", à Gennevilliers, $1^{\text {er }}$ avril 2015 @ Camille Millerand

\section{Évolutions récentes des accords}

Malgré des systèmes de protection sociale belge et français relativement proches, le contenu des accords bilatéraux, leur application ainsi que les modalités de réalisation de la portabilité des droits ont varié, dès leur origine. Tandis que, par exemple, les soins de santé couvraient l'ensemble des situations à l'exception des retraités en France, leur portabilité n'a été introduite que progressivement pour certaines catégories dans l'accord belgo-marocain. Le développement de la couverture de soins de santé au Maroc (AMO) a permis, en introduisant la possibilité d'une réciprocité du droit, une modification des accords bilatéraux en vue d'élargir la couverture aux retraités. Cette modification du système de Sécurité sociale marocain est à la base de la nouvelle convention française, entrée en vigueur en 2011, et de la négociation entamée entre la Belgique et le Maroc en vue d'en établir une nouvelle. La négociation autour de la refonte des accords bilatéraux dans les deux corridors migratoires s'est déroulée avec la triple ambition de moderniser les accords, de les adapter aux systèmes de Sécurité 
sociale respectifs, ainsi que de les homogénéiser entre eux. Ladaptation et la modernisation des accords se sont joués sur les plans de la modernisation des termes utilisés dans le corps du texte, d'une part, et dans l'introduction de nouveaux droits exportables en accord avec la transformation du système marocain, d'autre part. Les nouveaux accords introduisent la portabilité des soins de santé pour les retraités, augmentent le nombre d'enfants couverts par les allocations familiales et redéfinissent des règles de partitionnement de la pension de survie en cas de répudiation. Mais, outre des évolutions qui viennent adapter le contenu des accords aux systèmes de Sécurité sociale respectifs, on a pu assister à une réelle homogénéisation du contenu des accords bilatéraux.

Cette homogénéisation passe par la mise en conformité des accords avec la législation européenne au règlement (CEE) n¹408/71 du Conseil, du 14 juin 1971, relatif à L'adaptation et "l'application des régimes de la modernisation des accords se sont joués sur les plans

de la modernisation des termes utilisés dans le corps du texte, d'une part, et dans l'introduction de nouveaux droits exportables en accord avec la transformation du système marocain, d'autre part. Sécurité sociale aux travailleurs salariés, aux travailleurs non salariés et aux membres de leur famille qui se déplacent à l'intérieur de la Communauté" et au règlement $(C E) n^{\circ} 883 / 2004$ du Parlement européen et du Conseil du 29 avril 2004 portant sur la coordination des systèmes de Sécurité sociale. Ce dernier règlement vient, par ailleurs, rappeler la non-transportabilité des prestations non contributives au sein des États membres. L'accord belgo-marocain est relativement clair sur ce point, dans la mesure où de telles prestations n'ont jamais été transportables. Le cas français, plus complexe, va converger vers la réglementation européenne à la fin des années 2000. Le minimum vieillesse français était à l'époque divisé en deux types d'allocations (dites de premier et de deuxième étage), dont une partie (l'allocation de premier étage) était transportable ${ }^{13}$. Ce n'est plus le cas depuis 2010. De plus, la disparition du minimum vieillesse au profit de l'Aspa en 2007 vient régler le problème du contenu de la prestation.

\section{Les enjeux d'une comparaison internationale}

Deux lignes de tension émergent de ce que nous avons présenté. Premièrement, il y a une opposition entre réciprocité du droit et non-portabilité des prestations non contributives. Nous avons vu que la mise en place d'un système de soins de santé obligatoire au Maroc ainsi que la réforme du Code civil marocain, de façon plus limitée, ont été la cause de la refonte des accords bilatéraux dans les deux corridors. Néanmoins, cette refonte, soumise à des impératifs européens, a homogénéisé la nonportabilité des prestations non contributives dans le cas français. La réciprocité du droit ne peut donc s'appliquer sur ce type de prestation, ce qui peut poser problème compte tenu du niveau socio-économique des premières générations de migrants marocains et de leurs descendants en Belgique et en France.

Deuxièmement, on perçoit une tension entre une homogénéisation croissante des accords bilatéraux, d'une part, et des processus historiques particuliers, d'autre part. Cette tension est à lire sous la double optique de la convergence et de la "dépendance de sentier ${ }^{14 "}$. Convergence parce que l'introduction des soins de santé pour les retraités, la suppression de la transportabilité des minima sociaux, ainsi que l'élargissement du nombre d'enfants bénéficiaires d'une allocation familiale, sont des tendances qui marquent l'ensemble des accords bilatéraux renégociés au cours de la période et rendent comparables les accords belgo et franco-marocains. Dépendance de sentier parce que la France

13. Ilham Dkhissi, Jean-Marc Dupuis, Claire El Moudden, op. cit. 14. Jacques Wels, "La politique des fins de carrière. Vers un modèle européen convergent ?", in Sociologie, vol. 5, n 3, 2014, pp. 233-253. La “ dépendance de sentier" est une notion utilisée en sciences politiques et sociales, qui souligne l'influence des décisions qui ont été prises par le passé sur les décisions futures. 
et la Belgique n'entretiennent pas la même histoire avec le Maroc. Malgré un indéniable phénomène de convergence entre les pays, l'accord franco-marocain - du fait d'une histoire marquée par le protectorat français et d'un plus important nombre de Marocains émigrés en France - demeure plus large que l'accord belgo-marocain.

D'un point de vue empirique, la comparaison se heurte à deux problèmes majeurs : un problème de données et un problème de comparabilité des systèmes. Les statistiques ethniques ne font pas partie de la culture statistique de la France et, dans une moindre mesure, de la Belgique ${ }^{15}$. Les données, quand elles ne sont pas agrégées par continent d'origine, sont souvent indisponibles pour les descendants des immigrés ainsi que pour les individus ayant acquis la nationalité du pays. Il y a donc une nette difficulté à estimer combien de personnes et quel type de population migrent dans le cadre des accords bilatéraux. Cette limite est accentuée par la difficulté de suivre les populations dans l'espace. Compte tenu du faible nombre de migrants, les bases de données internationales de type SHARE, EFT, ESS ne permettent pas de suivre le profil statistique des migrants. De même, les bases de données administratives nationales ne permettent pas ce type de suivi. Mais il y a également difficulté à comparer des systèmes de Sécurité sociale différents. Le cas des minima sociaux est en cela révélateur. Des dispositifs tels que la Grapa ou l'Aspa n'existent pas dans tous les pays. L'organisation, selon des bases différentes, des systèmes de Sécurité sociale rend difficile la correspondance entre les pays et exige d'envisager des processus de transportabilité adaptés à une réciprocité qui n'est pas toujours mimétique. Si les minima sociaux ne sont pas transportables, la pension de survie dont nous avons parlé plus haut offre une réciprocité du droit dans les trois pays concernés, mais le contenu asymétrique des codes civils respectifs rend complexes les modalités de portabilité du droit. Dans de tels cas, la solution fournie par les accords bilatéraux peut être adaptée par décision de justice. Il y a donc, dans ce cas spécifique, individualisation du droit en fonction de critères qui font jurisprudence. L'enjeu, à l'avenir, sera - dans un monde soumis à un flux migratoire croissant et à des parcours entre des territoires multiples - de garantir une portabilité du droit qui soit adaptée aux situations individuelles sans être pour autant individualisée. 